\title{
Rayleigh-Taylor instability after giant impacts: Imperfect equilibration of the Hf-W system and its effect on the core formation age
}

\author{
Takanori Sasaki and Yutaka Abe \\ Department of Earth and Planetary Science, University of Tokyo, 7-3-1 Hongo, Bunkyo-ku, Tokyo 113-0033, Japan
}

(Received April 4, 2006; Revised February 20, 2007; Accepted July 3, 2007; Online published September 28, 2007)

\begin{abstract}
Hf-W chronometry provides constraints on the timing of planetary accretion and differentiation, as the segregation of a metal core from silicates should induce strong fractionation of $\mathrm{Hf}$ from W. In most previous studies, it was assumed that a giant impact would perfectly reset the Hf-W chronometer. Here, we show the difficulty of achieving perfect equilibration of the Hf-W system. Perfect equilibration requires iron to split into small droplets. However, since the sedimentation velocities of small droplets are low, the Rayleigh-Taylor instability between the upper metal-containing and lower metal-free layers results in quick overturning of the layers, unless iron droplets were uniformly distributed in the entire mantle. Therefore, the lower metal-free layers cannot be equilibrated. We calculated the isotopic evolution of the Hf-W system, taking into account the partial resetting of this chronometer. Our study led to three conclusions: (1) collision conditions and the number of giant impact events affect the age estimation of core formation, (2) the Earth's W isotope ratio indicates that more than two-tenths of the volume of the protoearth's mantle must have been equilibrated at each giant impact, and (3) Mars should have experienced a late, extensive equilibration event; it could have been a single giant impact.
\end{abstract}

Key words: Accretion, collisional physics, Earth, geochemistry, planetary formation.

\section{Introduction}

Hafnium and tungsten are both highly refractory elements; hafnium is a lithophile element, whereas tungsten is a moderately siderophile element that should partition strongly into the metal phase during metal/silicate segregation. The decay of now extinct ${ }^{182} \mathrm{Hf}$ (half-life, $9 \mathrm{Myr}$ ) to ${ }^{182} \mathrm{~W}$ is an ideal chronometer for tracing core formation events, because $\mathrm{Hf}$ is retained in the silicate mantle while $\mathrm{W}$ is largely partitioned into the core during core segregation (Harper et al., 1991; Lee and Halliday, 1995; Harper and Jacobsen, 1996).

It has been argued that the age of terrestrial core formation was limited by the accretion time and that it could have occurred $60 \mathrm{Myr}$ later than the formation of iron meteorites (Lee and Halliday, 1995, 1996). Based on new measurements of the $\mathrm{W}$ isotope compositions and $\mathrm{Hf} / \mathrm{W}$ ratios of several meteorites, the age of terrestrial core formation and Earth's accretion has recently been re-estimated by some groups. These estimations were based on either a magma ocean model or a two-stage model (Harper and Jacobsen, 1996; Jacobsen, 1988). The magma ocean model assumes that growth of the Earth occurred at an exponentially decreasing accretion rate and does not consider the effects of giant impacts. The main growth stage for the Earth as defined in the magma ocean model of Yin et al. (2002), which corresponds to $63 \%$ growth, was estimated to occur at about $10 \mathrm{Myr}$. In this model, the end of Earth's accretion would have occurred at about 55 Myr (Kleine et al., 2004a). On

Copyright (c) The Society of Geomagnetism and Earth, Planetary and Space Sciences (SGEPSS); The Seismological Society of Japan; The Volcanological Society of Japan; The Geodetic Society of Japan; The Japanese Society for Planetary Sciences; TERRAPUB the other hand, the two-stage model assumes that Earth's core formed at a well-defined point in time by a single event (e.g. a giant impact). This model age does not provide a realistic age for core formation, but it does provide the earliest time when core formation can have ceased (Halliday et al., 1996; Halliday, 2004; Kleine et al., 2004a). In this model, the Earth's core formation would have ceased at about 30 Myr (Kleine et al., 2002; Schönberg et al., 2002; Yin et al., 2002).

Some studies have considered the effect of partial resetting of the Hf-W chronometer (Jacobsen and Harper, 1996; Sasaki and Abe, 2003; Halliday, 2004; Kleine et al., 2004a; Jacobsen, 2005; Nimmo and Agnor, 2006). These studies have suggested that without a quantitative assessment of the equilibration process resulting from giant impacts, we cannot use Hf-W chronometry to determine accurately the age of a giant impact or the age of metal-silicate separation. Meanwhile, Kleine et al. (2004a) derived constraints for the degree of metal-silicate equilibration during Earth's accretion by use of the ${ }^{182} \mathrm{~W}$ excess of the Earth's mantle relative to chondrites conversely.

In this paper, we first discuss the mechanisms that can cause imperfect equilibration of Hf-W systems as a result of giant impact events. We then discuss how Hf-W age measurements are affected by imperfect resetting. Finally, we discuss the core formation events on Earth and Mars.

\section{Mechanisms of Imperfect Equilibration of Hf- W Systems}

A giant impact can produce a global magma ocean on a protoearth, which, on first glance, seems to lead to complete metal-silicate equilibration as a direct result of the giant 
impact, unless the impact leads to direct core merger. Rubie et al. (2003) concluded that small metal droplets are formed and that their settling through the magma ocean can achieve chemical equilibration between metal droplets and silicate liquid. In this section, however, we point to a difficulty in achieving perfect equilibration in Hf-W systems, even if small droplets are formed, as suggested by Rubie et al. (2003).

\subsection{Achievable resetting ratio by the Stokes sedimen- tation of metal grains}

To achieve perfect equilibration of the Hf-W system in a mantle with added metal on the Earth's surface, this metal must split into small metal droplets and sink through the mantle in order to equilibrate with the surrounding mantle. There is isotopic and elemental exchange between the sinking metal droplets and silicate melts to maintain the distribution of $\mathrm{W}$ between metal and silicate, as defined by the metal-silicate partition coefficient. The degree of equilibration of the Hf-W system in the mantle depends on the total surface area of metal droplets. The smaller the metal droplets, the easier the mantle can be equilibrated. First, we estimated the required size of these droplets for complete metal-silicate equilibration. In the following calculations, we used the same physical properties (diffusivity and viscosity in the magma ocean) as Rubie et al. (2003).

During the stage of planetary accretion, newly accreted $\mathrm{W}$ in the impactor's metal component equilibrates with some portion of the $\mathrm{W}$ in the silicate melt of the target and leads to a Hf/W ratio for this portion corresponding to the value to meet the metal-silicate partition coefficient for a mixture between impactor metal and target silicate. This equilibration continues during the sedimentation of metal in the silicate mantle. Suppose that metal in the impactor splits into lots of metal spheres with radius $r$ sinking at the Stokes velocity:

$$
v_{s}=\frac{2 \Delta \rho_{s} g r^{2}}{9 \eta}
$$

where $\Delta \rho_{s}$ is the difference in density between the silicate melt and metal, and $g$ is the gravitational acceleration. Typical values are $\Delta \rho_{s}=8 \cdot 10^{3} \mathrm{~kg} / \mathrm{m}^{3}$ and $g=10 \mathrm{~m} / \mathrm{s}^{2}$. The viscosity of the silicate melt $\eta$ is $10^{-2} \mathrm{~m} \cdot \mathrm{s} / \mathrm{kg}$ (Rubie et al., 2003), assuming a completely molten global magma ocean for the Earth's mantle. We can estimate the equilibrated volume from the metal spheres:

$$
v_{\mathrm{eq}}=2 \pi r \sqrt{D \tau} H, \quad \tau=\frac{2 r}{v_{s}}
$$

where $D$ is the diffusivity of $\mathrm{W}$ in the silicate melt, $D=$ $10^{-8}$ (Rubie et al., 2003), and $H$ is the depth of the magma ocean in the target body. We adopt the present-day depth of Earth's mantle, $H=2.9 \times 10^{6} \mathrm{~m}$. The volume of mantle in which the equilibration of the Hf-W system takes places at a single giant impact, $V_{\text {eq }}$, is given by the sum of $v_{\text {eq }}$ over all of the iron particles. We define the resetting ratio of the HfW chronometer to be the ratio of $V_{\text {eq }}$ and the total volume of the target mantle and impactor mantle.

First, assuming that the metal in the impactor breaks into small metal spheres of equal size, we can easily estimate the
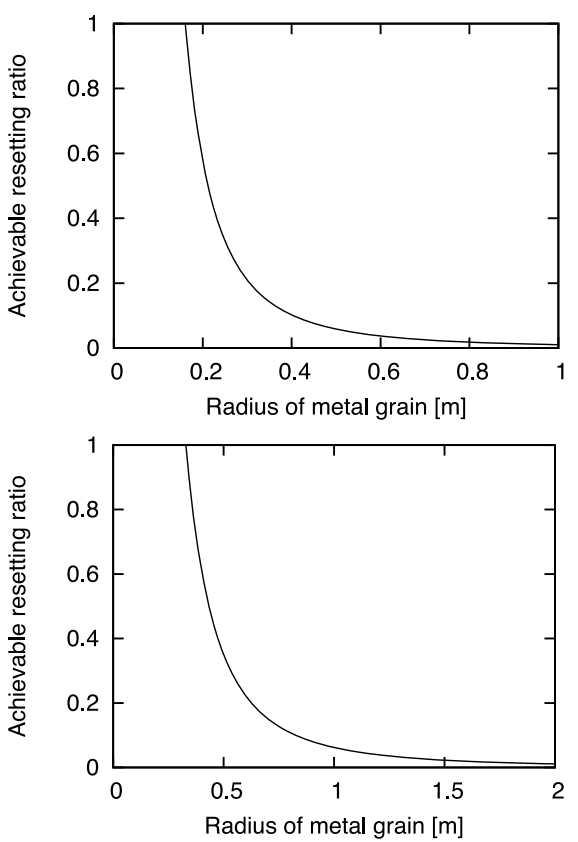

Fig. 1. The resetting ratio achievable as a function of the radius of fragmented metals. We consider those cases in which the metals from the impactor break into small fragments of equal size (upper), with a size distribution of metal spheres given by a power law (lower).

resetting ratio of the Hf-W chronometer. As shown in Fig. 1 (upper), the radius of metal spheres must be less than about $20 \mathrm{~cm}$ to achieve perfect equilibration of the Hf-W system. In this estimation, the target body was assumed to be the size of the Earth, and the impactor body was assumed to be the size of Mars, with the appropriate core sizes for each object.

Next, suppose that the size distribution of metal spheres is given by a power law. We can estimate $V_{\text {eq }}$ as a function of the radius of the largest metal sphere:

$$
\begin{aligned}
& V_{\mathrm{eq}}=\int_{m}^{M} 2 \pi r \sqrt{D \tau} H f(m) d m, \\
& f(m)=\frac{2-b}{M^{2}} M_{0}\left(\frac{m}{M}\right)^{-b}
\end{aligned}
$$

where $M$ is the maximum mass of metal spheres and $M_{0}$ is the mass of the impactor's core. The cumulative power law index $b$ is known to be close to 1 for the impact fragments (Oddershede et al., 1993). When $b=1$ in Eq. (3), the radius of the largest metal sphere should be less than about $40 \mathrm{~cm}$ to achieve perfect equilibration of the Hf-W system (Fig. 1, lower).

In these estimations, we assumed that the metal and silicate portions of impactors were well mixed at the impact and placed upon the top of Earth's mantle with iron split into spheres of given size. Any part of the silicate composition through which the metal spheres passed was equilibrated. We also assumed all metal accreted to the Earth was involved in the metal-silicate equilibration. This gives an extreme case of equilibration which is independent of the Hf/W history and the initial $\mathrm{W}$ composition of the impactor. For these reasons, Fig. 1 shows the upper limits of the achievable resetting ratio for a given size of metal droplets. 


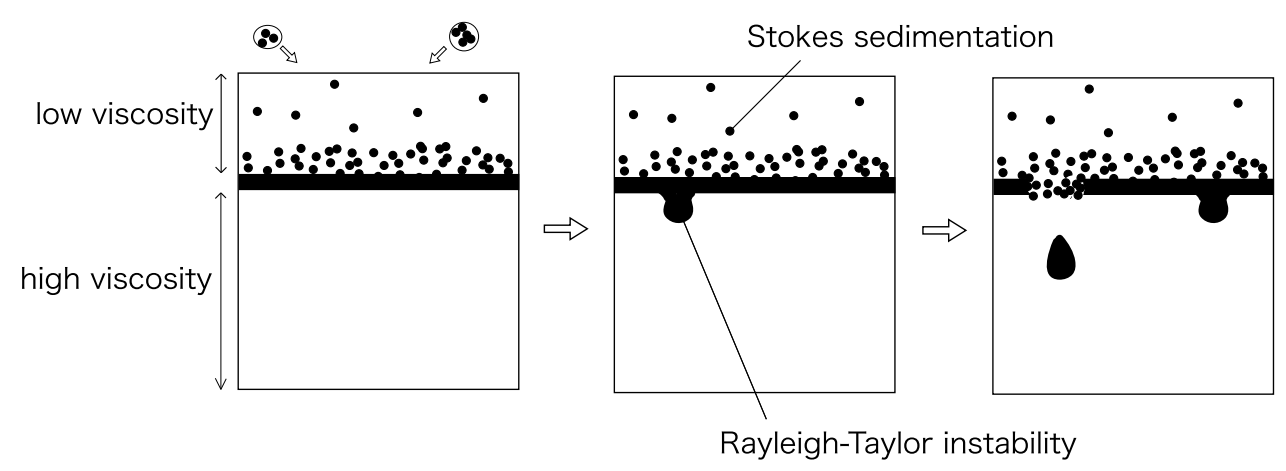

Fig. 2. Sketch of the metal droplets' behavior in the silicate melt layer, which is assumed to have a low viscosity in the upper layer and high viscosity in the lower layer. Infalling ejecta and impact fragments descend in the low-viscosity upper layer at the Stokes velocity and are deposited over the lower layer. The Rayleigh-Taylor instability then results in big blobs of metallic material sinking into the core.

\subsection{The Rayleigh-Taylor instability and the Earth's core formation}

As discussed in the previous section, the metal droplets in the mantle must be centimeter-scale in size to achieve perfect equilibration of the Hf-W system. In addition, the droplets must descend in the mantle from the surface to the core without producing large blobs or metal layers to achieve perfect equilibration.

Many recent works have discussed the modeling of giant impacts using a method known as smooth particle hydrodynamics, or SPH (e.g., Canup and Asphaug, 2001; Canup, 2004). These results show that if an impactor and most parts of the target break into pieces, then the debris mixes during the re-accretion onto the surface of the protoearth. Jacobsen (2005) observed that these SPH simulations suggest accretion with re-equilibration with the impactor and the target mantle. However, these simulations with $\mathrm{N}=10^{4}-$ $10^{5}$ particles, implying particle sizes of about $10^{2} \mathrm{~km}$, do not have enough resolution to consider sizes smaller than meter-scale. Moreover, to date, no simulations that demonstrate how the target's mantle breaks and mixes with the impactors in giant impacts exist. Therefore, it is not possible to discuss the metal-silicate equilibration based on these SPH simulations.

Although a protoearth could be undifferentiated before the first giant impact (i.e. iron droplets are distributed entire mantle uniformly), it would become differentiated after the giant impact (i.e. most metal would fall into the core). Giant impacts are considered to have occurred more than once from the physically (Kokubo and Ida, 1998) and geochemically (Kleine et al., 2004a) point of view. Therefore, we do not consider the case that the iron droplets are distributed in the protoearth's entire mantle in an undifferentiated state before all giant impacts. Since giant impacts are expected to occur at long intervals, the target body's metal component after an impact is assumed to fall into the core before the next giant impact occurs, and so the target's mantle is assumed to hold no metal at the time of each giant impact. On the other hand, the impactor's metal component and mantle are assumed to break into pieces as a result of the giant impact, so that infalling debris from the impactor is assumed to be a mixture of metal and silicate. SPH simulations for a head-on impact case, that is, a low-angular-momentum collision case, suggest the possibility that the impactor's core could sink into the target's core without breaking (Cameron, 1997). However, because the mantle clearly cannot be equilibrated in this case, the assumption of the break-up of the impactor's core and mixing of metal and silicate gives us the higher limit of equilibration degree. Moreover, SPH simulations have generally shown that while some of the impactor's core may diffuse into the target's mantle, much of the impactor's core would be ejected and fall back to the Earth later (Cameron, 2000), so that not all of the impactor's metal could be distributed in the entire mantle initially. Therefore, we assume that the iron droplets are distributed not in the entire mantle but only in the upper layer of the mantle after the giant impact. These assumptions are the initial conditions for our consideration.

In this section, we consider two possible scenarios for the structure resulting from giant impacts: (1) two layers are produced, a fully molten layer (upper layer) and a partially molten layer (lower layer); (2) the entire mantle becomes fully molten.

2.2.1 A fully molten layer (upper layer) and a partially molten layer (lower layer) are produced In the upper molten layer, the infalling debris of the impactor and the broken target would be easily separated into metal and silicate. Metal droplets would then descend in the upper molten layer and deposit over the lower viscous layer. When the thickness of the accumulated metal layer exceeds a critical value, the Rayleigh-Taylor instability becomes important. Metallic materials would then fall through the lower layer into the central part of the planet (Elsasser, 1963; Stevenson, 1981; Sasaki and Nakazawa, 1986) (Fig. 2) in the form of kilometer-sized blobs. Since the size of such metal blobs would be too large to achieve perfect equilibration of the Hf-W system (Fig. 1), the lower layer of the mantle, at least, could not be equilibrated. Therefore, in this scenario, irrespective of the initial size of the impactor's falling metal droplets, the lower mantle cannot be equilibrated.

2.2.2 The entire mantle is fully molten If an impactor's core breaks into droplets, they will be larger than meter-scale at the impact, and the sinking of these metal droplets, therefore, cannot equilibrate the whole mantle, as shown in the Fig. 1. However, if these droplets can break up into smaller droplets in the molten mantle, there is a chance of perfect equilibration. Rubie et al. (2003) concluded that 


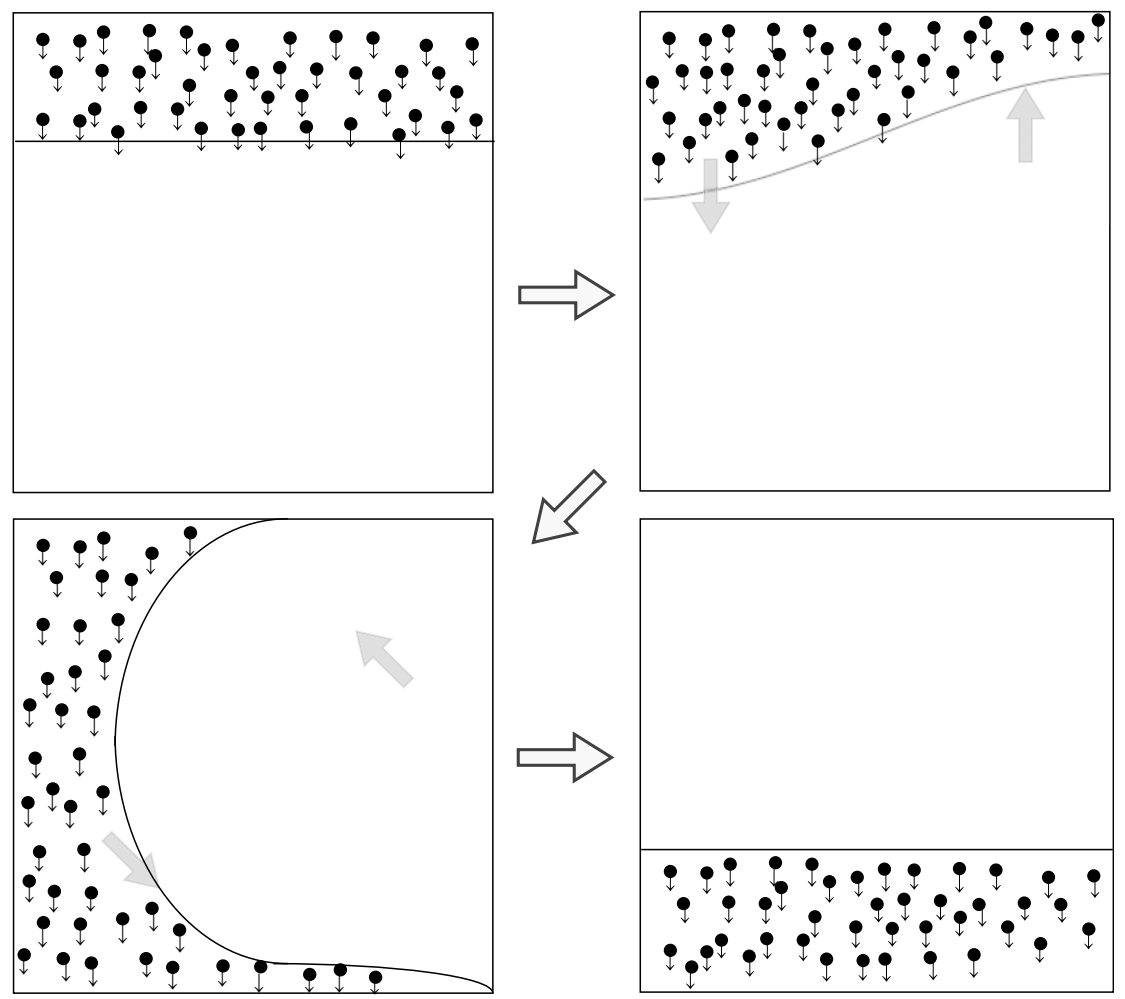

Fig. 3. Sketch of the metal droplets' behavior in the silicate melt layer, which is assumed to be fully molten. After a giant impact, the ejecta and broken impactor are mixed with each other and accrete to the protoearth as a mixture. The accreted mixture is separated into silicate and centimeter-scale metal droplets, and then these droplets sink into a well-mixed metal-silicate layer (first sketch). If these droplets are small enough to achieve perfect equilibration, the Rayleigh-Taylor instability between the silicate melt layer with metal droplets and the metal-free layer occurs immediately because the growth of the Rayleigh-Taylor instability is much faster than the Stokes sedimentation of metal droplets (second sketch). Once the Rayleigh-Taylor instability has grown, the layer with metal droplets sink into the core as a cluster, and so the mixture layer and metal-free layer overturn immediately (third sketch). In this situation, the mixture layer that holds small metal droplets- the upper layer in the first sketch-has an equilibrated Hf-W system. However, the metal droplets in the mixture layer cannot interact with silicate outside the cluster. Thus, there exist two regions in the mantle: an equilibrated region (last sketch, lower layer) and a non-equilibrated region (last sketch, upper layer).

the metal could equilibrate with silicate melt in a magma ocean because the molten metal dispersed as small droplets. They considered the idea that the small metal droplets could sink like "rainfall droplets" without forming big blobs and that the silicate layer could achieve equilibration of the HfW system by the Stokes sedimentation of these small metal droplets.

However, even in such a situation, we point out that there is the possibility of another type of Rayleigh-Taylor instability between the silicate melt layer with metal droplets and the silicate melt layer that is metal-free (Kobayashi et al., 1993) (Fig. 3). The Rayleigh-Taylor instability can occur between the upper mixture layer and the lower molten silicate layer because the upper layer mixed with metal droplets has a higher density than the lower metal-free layer. If the growth of the instability is much faster than the sedimentation of small iron droplets in the molten mantle, then the overturn of metal-rich and metal-free layers should occur. In this case, the metal-free layers cannot be equilibrated. This type of Rayleigh-Taylor instability is actually observed in laboratory experiments (Iga and Kimura, 1993).

In the case where an impactor's core initially breaks into droplets small enough to achieve perfect equilibration, such metal droplets would tend to stay at the top of the molten mantle because the sedimentation velocity of small droplets is very small, as we show in Subsection 2.3. Therefore, in this scenario it is also likely that the upper silicate layer contains many small metal droplets, and the Rayleigh-Taylor instability between the upper and the lower layer should occur as depicted in Fig. 3.

\subsection{Timescale comparison between Stokes sedimenta- tion and the Rayleigh-Taylor instability}

When any mass of dense liquid (molten metal) falls through a less dense liquid (molten mantle), instabilities tend to form at the interface that may eventually disperse the dense mass into small droplets (Rubie et al., 2003). In this section, we consider the consequences of the assumption that the metals break up into small grains in the magma ocean. We assumed a mixture layer of metal and silicate on a metal-free layer. Since metal droplets are small, we assume equilibrium is achieved in the mixture layer. However, whether or not equilibration of the whole mantle occurs can be assessed by comparing the timescales between the Stokes sedimentation and growth of the Rayleigh-Taylor instability at the interface of the metal-silicate mixture layer and the metal-free silicate melt layer. In this paper, we consider two extreme cases: (1) the thick limit and (2) the thin limit of the mixture layer (see Appendix).

Figure 4 shows that timescale ratios (growth of the Rayleigh-Taylor/Stokes sedimentation) as a function of the sinking depth of metal grains by Stokes sedimentation. In both cases, the timescale of the growth of the Rayleigh- 


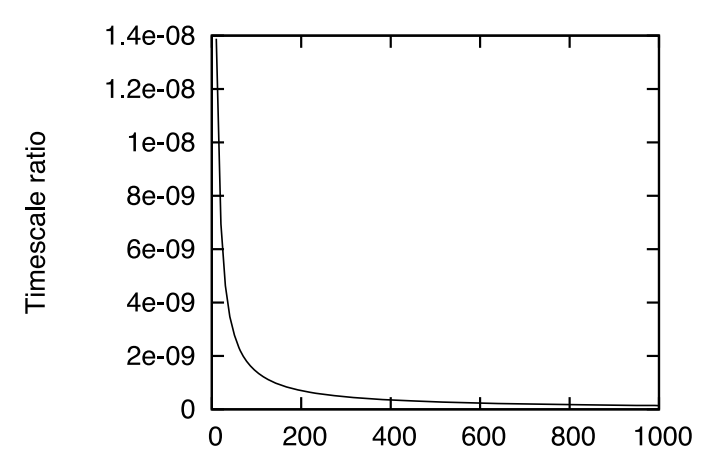

Sink depth by the Stokes sedimentation [m]

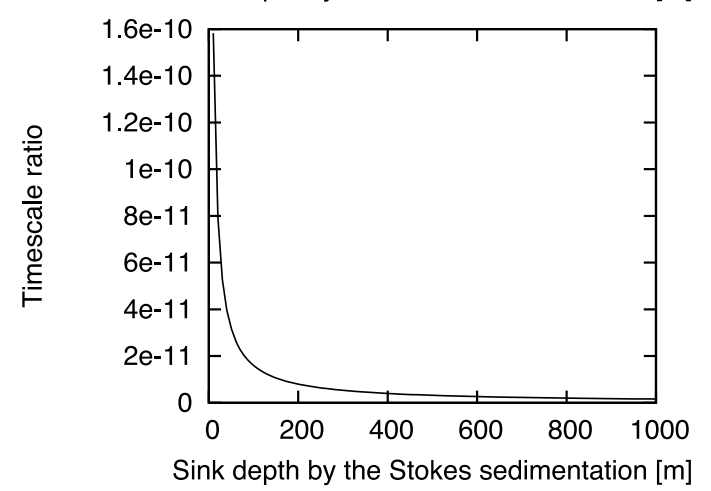

Fig. 4. Timescale ratios between growth of the Rayleigh-Taylor instability and Stokes sedimentation as a function of the sinking depth by Stokes sedimentation. The upper panel (1) shows an example of a thick mixture layer, and the lower panel (2) the case of a thin mixture layer. The radius of metal grains is $1 \mathrm{~cm}$ in both cases.

Taylor instability is much shorter than that of the Stokes sedimentation. Therefore, the Rayleigh-Taylor instability should grow quickly, before metal grains can descend significantly through the mantle. Once the Rayleigh-Taylor instability has grown, the mixing layer should fall or overturn into the core as a cluster.

So far, we have estimated the timescale using a twodimensional rectangle box. It is also important to check the validity of applying the results to spherical shells. In fact, the linear stability analysis of the problem yields the same results in two or three dimensions, and growth rates obtained through a spherical harmonic expansion are independent of azimuthal wavenumber (Goodman, 1990). It has also been confirmed that the dominant wavelength of the instability will remain the same in three dimensions (Müller et al., 1991).

\subsection{Summary}

After a giant impact, if two layers (low-viscosity upper layer and high-viscosity lower layer) are formed, then the metal grains will descend, deposit over the lower layer, and then fall through the lower layer as a large blob produced by the Rayleigh-Taylor instability between the metal layer and the high-viscosity lower silicate layer (Fig. 2). In this case, therefore, perfect equilibration of the Hf-W system cannot be achieved.

If all of the mantle is rendered fully molten by a giant impact, and the falling impactor's metal debris has split into small droplets (Rubie et al., 2003), these droplets can descend through the molten mantle. Then, if the mantle and metal droplets in the metal-containing layer are mixed well, this layer can be equilibrated completely. However, even in this situation, the Rayleigh-Taylor instability acts between the metal-containing layer and the metal-free layer to cause the overturn of these two layers (Fig. 3), so that these droplets cannot continue to descend to the bottom like "rainfall droplets" and cannot equilibrate the entire mantle. Thus, although the metal-containing layer can be equilibrated, the metal-free layer cannot be equilibrated for the Hf-W system. Therefore, the complete metal-silicate equilibration by means of a giant impact cannot be expected in this case either.

Jacobsen (2005) suggested that giant impacts would completely reset the Hf-W chronometer in many cases. Although it is based on the observation of SPH simulations, the resolution of the models is still too coarse to be conclusive, as noted above. The consideration of the RayleighTaylor instability, to the contrary, leads to the conclusion that perfect resetting occurs only in very limited cases; perfect equilibration is possible only when the iron droplets are smaller than meter-scale and are distributed throughout the entire mantle in the first place, without a metal free layer being produced. However, such a distribution of metal droplets is unlikely, in particular for repeated giant impacts, as noted above. Thus, we conclude that it is difficult to obtain complete equilibration of the Hf-W system during giant impacts.

The occurrence of the Rayleigh-Taylor instability is not limited to the core formation after giant impacts considered here. If a metal-free layer exists below a newly accretediron-silicate mixture, the Rayleigh-Taylor instability likely occurs, and silicates in the metal free layer would not be equilibrated with iron. Thus, complete metal-silicate equilibration also seems to be difficult in most case of core formation other than that after giant impacts.

\section{Isotopic Evolution of the Hf-W System in the Case of Imperfect Equilibration}

In this section, we calculate the isotopic evolution of the Hf-W chronometer in the case of imperfect equilibration of the Hf-W system. We focus attention on deriving required conditions (not sufficient conditions) to meet the observational data for each equilibrating event. As such, we consider cases for which the equilibration between metal and silicate occurs most easily. We use assumptions and simplifications which give us a higher limit of the resetting ratio of each equilibrating event. Although such assumptions may not be realistic, we can provide a number of required conditions for each equilibrating event so as to obtain the observed isotopic ratio.

The simplest model of fractionation is a two-stage model with a single episode of core formation occurring at $t=$ $t_{\mathrm{cf}}$, where $t$ is defined with time running forward from the origin of the solar system, $T_{0}$ years ago. The isotope time evolution of reservoir $j$ is given by

$$
\begin{aligned}
\epsilon\left(t_{\mathrm{cf}}, t_{\mathrm{obs}}\right)= & Q \cdot f_{j} \cdot\left(\frac{{ }^{182} \mathrm{Hf}}{{ }^{180} \mathrm{Hf}}\right)_{0} \\
& \cdot\left[e^{-\lambda_{182} t_{\mathrm{cf}}}-e^{-\lambda_{182} t_{\mathrm{obs}}}\right]
\end{aligned}
$$

where $t_{\mathrm{obs}}=T_{0}$ yields the present value (Harper and Jacobsen, 1996). Isotopic evolution relative to the CHondritic 
Uniform Reservoir (CHUR) began after $t_{\mathrm{cf}}$ years from the initial state. In the above equations $\lambda_{182}$ is the ${ }^{182} \mathrm{Hf}$ decay constant (0.0779 $\mathrm{Myr}^{-1}$ (Vockenhuber et al., 2004)), and $Q=10^{4} \cdot\left({ }^{180} \mathrm{Hf} /{ }^{182} \mathrm{~W}\right)_{\mathrm{CHUR}}=1.49 \times 10^{4}$ (Kleine et al., 2004b). The Hf/W fractionation in a reservoir $j$ relative to CHUR is defined by the $f$-value:

$$
f_{j}=\frac{\left({ }^{180} \mathrm{Hf} /{ }^{183} \mathrm{~W}\right)_{j}}{\left({ }^{180} \mathrm{Hf} /{ }^{183} \mathrm{~W}\right)_{\mathrm{CHUR}}}-1
$$

We use values $f_{j}=12$ for the Earth's region (Harper et al., 1991; Yin et al., 2002) and $\left(\frac{{ }^{182} \mathrm{Hf}}{{ }^{180} \mathrm{Hf}}\right)_{0}=1 \times 10^{-4}$ (Kleine et al., 2002; Schönberg et al., 2002; Yin et al., 2002; Kleine et al., 2005) from a survey of estimates of Hf/W ratios in the silicate Earth. In fact, there is an uncertainty in the $f$-value, and estimates range from 10 to 40 (Newsom et al., 1996). The effects of varying the $f$-value on our conclusions are discussed at the end of this section.

Recent studies on planetary formation have shown that several tens of Mars-sized protoplanets are formed through the successive accretion of planetesimals in the terrestrial planet region. Then, during the final stages of planetary formation, the protoearth suffers collisions with other protoplanetary bodies, known as giant impacts (Kokubo and Ida, 1998; Wetherill and Stewart, 1989). We therefore consider two different stages: the protoplanet formation stage and the giant impact stage.

In the protoplanet formation stage, through each impact of a planetesimal, a part of the target body is equilibrated, and the $\epsilon$ value of this part is reset to the initial value. All of the impactor's mantle is assumed to be equilibrated during the impact, and each impact equilibrates the target's mantle by $k$ times the impactor's volume, $d V$. The degree of equilibration, $k$, resulting from each planetesimal impact is a parameter in this model. Thus, each impact equilibrates the target mantle in a volume $k \cdot d V$. Therefore, the $n$th impact, occurring at $t=t_{n}$, equilibrates a fraction $k \cdot \frac{d V}{V_{n}}$ of the target mantle, where $V_{n}$ is the volume of the target body at the time of the $n$th impact, and updates the $\epsilon$ value to $\epsilon\left(t_{n}, T_{0}\right)$ in the equilibrated region. Meanwhile, the rest of the target mantle, of fractional volume $\left(1-k \cdot \frac{d V}{V_{n}}\right)$, retains its value at the time of the $(n-1)$ th impact. In this calculation, for simplicity, we assumed that the whole mantle is well mixed after each impact and that the $\epsilon$ value for any part of the mantle has the average $\epsilon$ value of the whole mantle at the time of each impact. Therefore, the isotopic fractionation by $n$th impact is given by

$$
\epsilon_{n}=k \cdot \frac{d V}{V_{n}} \cdot \epsilon\left(t_{n}, T_{0}\right)+\left(1-k \cdot \frac{d V}{V_{n}}\right) \cdot \epsilon_{n-1}
$$

where $\epsilon\left(t_{n}, T_{0}\right)$ is given by Eq. (4). We iterated this calculation for each planetesimal impact a hundred million times at regular time intervals before the formation of protoplanets. In other words, we assumed that the planetesimal accretion rate was constant in this calculation. Here we define the age of protoplanet formation as the age of the end of the first stage, or the end of planetesimal accretion to protoplanet. A plot of $\epsilon$ versus the age of protoplanet formation is shown in Fig. 5 . $k$ is varied from 0.1 to 10 ; that is, each impact equilibrated the target's mantle by 0.1 to 10 times the impactor's

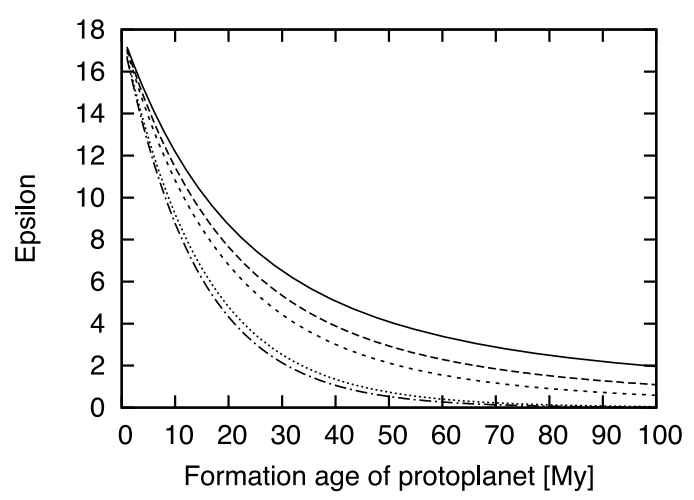

Fig. 5. Isotopic evolution of $\epsilon$ as a function of protoplanet formation age in the Earth's region. Parameter $k$ [Eq. (6)] is 0.1, 0.5, 1, 5, 10 from top to bottom.

volume. This results in $\epsilon$ values of 10-12 at $10 \mathrm{Myr}$ (Fig. 5). During this stage, the parameter $k$ does not greatly affect the isotopic fractionation until the formation of a protoplanet at $10 \mathrm{Myr}$, i.e., a factor of 100 difference in the parameter $k$ results in an $\epsilon$ difference of only 2 . Therefore, during this stage, the Hf-W system's isotopic evolution does not play an important role, as long as it is assumed that protoplanet formation was completed in less than $10 \mathrm{Myr}$ (Kokubo and Ida, 2000).

In the giant impact stage, each giant impact partially equilibrates the target body and, thus, partially resets its chronometer. We define the resetting ratio as the ratio of equilibrated volume of mantle. The resetting ratio in relation to a giant impact, $p$, and the number of giant impacts, $n$, are parameters in this model. All of the impactor's mantle was assumed to be equilibrated by the giant impact for simplicity, which leads to a larger estimation of the equilibration rate of the Hf-W system in our calculation. This assumption also means that the $\mathrm{W}$ isotope composition of the impactor mantle is reset at giant impact and that the results are independent of the Hf-W history of the impactor. This simplification gives us a lower limit of the required resetting ratio of giant impact, which is discussed at the end of this section. Each giant impact is assumed to equilibrate a fraction $p$ of the target's mantle. We also assumed that the whole mantle is well mixed after each giant impact. The $\epsilon$ value of the protoplanet, which was experienced during the $n$th giant impact at a time $t_{n g}$, is given by

$$
\epsilon_{n}=p \cdot \epsilon\left(t_{n g}, T_{0}\right)+(1-p) \cdot \epsilon_{n-1}
$$

We used the $\epsilon$ value shown in Fig. 5 for the initial $\epsilon$ value at the first giant impact. We assumed that the first giant impact occurred at $10 \mathrm{Myr}$, so the initial value of $\epsilon$ was fixed at 10, according to the result of Fig. 5. Subsequent giant impacts were assumed to occur at regular intervals. The Earth's mantle has a $\epsilon$ value of 0 and chondrites have a value of -1.9 , which means that the Earth's mantle has an excess of about $2 \epsilon$ units (Kleine et al., 2002, 2004a; Schönberg et al., 2002; Yin et al., 2002). We calculated the age of the last giant impact satisfying this Earth's observational data as a function of the resetting ratio of each giant impact. In our calculations using Eq. (6) and Eq. (7), the entire Earth's core was assumed to be involved in the 


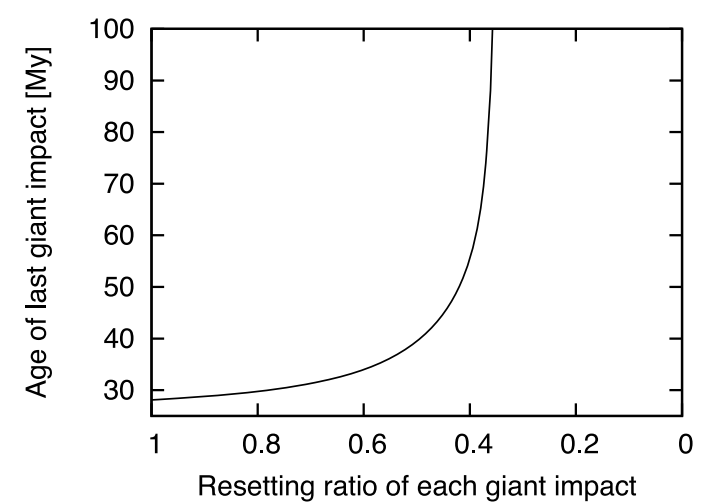

Fig. 6. The age of the last giant impact as a function of the resetting ratio of each giant impact, fitting to the observational data $(\epsilon=2)$ from Earth samples. The number of giant impacts is assumed to be five. The initial state is $\epsilon=10$ at $t=10$. The formation age of the Earth for perfect resetting (resetting ratio $=1$ ) is about $30 \mathrm{Myr}$, in agreement with a previous study (Yin et al., 2002).

metal-silicate equilibration. This would not be a realistic assumption. However, these calculations give us the higher limit of equilibration at each giant impact event. Therefore, from the viewpoint of obtaining the observed isotopic ratio, we can obtain the lower limit of the resetting ratio required for each giant impact. That is, we use assumptions that lead to the "higher limit of equilibration" on calculating isotopic evolution to obtain "the lower limit of required resetting ratio" to meet the observed epsilon value. The number of giant impacts, $n$, was varied from 2 to 10 . Figure 6 shows the result for $n=5$, for example. The estimated age of the last giant impact depends on the resetting ratio of each giant impact, which must be greater than 0.3 to yield the observed $\epsilon$ value. The effect of the number of giant impacts is shown in Fig. 7. It shows that the resetting ratio of each giant impact and the number of giant impacts both affect the estimation of the age of the last giant impact. The results indicate that the average resetting ratio of each giant impact must be greater than 0.2 to yield a good fit with the observations, even if giant impacts occurred ten times.

Although we use the $f$-value of 12 in Eq. (4) to solve Eq. (6) and Eq. (7), as mentioned earlier, the $f$-value in Eq. (4) is considerably uncertain (from 10 to 40). Therefore, we check how does this uncertainty affect our conclusions here. Figure 8 shows the age of last giant impact assumed to have occurred ten times to form the Earth for $f=10$, $12,20,30,40$, which was calculated in a manner similar to the case of $f=12$. It shows that the lower limit of average resetting ratio of each giant impact is still about 0.2 in the range from $f=10$ to $f=40$, which would not alter our conclusions.

Our calculations tend to give an overestimation of the equilibration rate of the Hf-W system, as all of the impactor's core and mantle is assumed to be equilibrated by a giant impact. In practice, because some fraction of the impactor's core (mantle) may be added to the target's core (mantle) without equilibrating, the required resetting ratio may be larger than that was estimated in this section. Therefore, our value of 0.2 should be regarded as a lower limit of the required resetting ratio of each giant impact, for a total

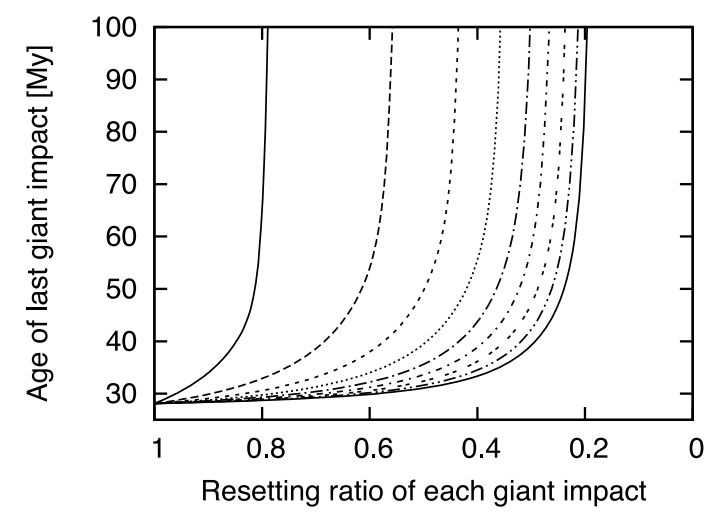

Fig. 7. The age of the last giant impact as a function of the resetting ratio of each giant impact, fitting to the observational data $(\epsilon=2)$ from Earth samples. The number of giant impacts is 2 to 10 from left to right. The initial state is $\epsilon=10$ at $t=10$.

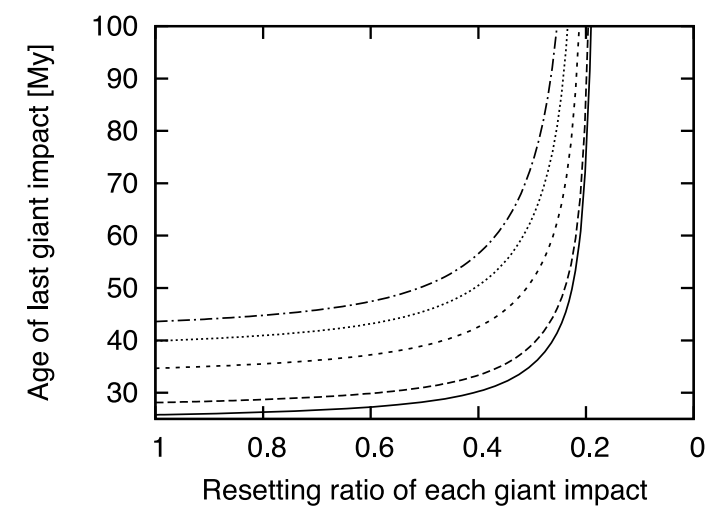

Fig. 8. The age of the tenth giant impact as a function of the resetting ratio of each giant impact, fitting to the observational data $(\epsilon=2)$ from Earth samples. The $f$-value is $10,12,20,30,40$ from bottom to top. Each initial $\epsilon$-value was calculated using Eq. (6) and Eq. (7) for each $f$-value.

number of giant impacts less than or equal to ten.

Nimmo and Agnor (2006) considered two extreme scenarios after giant impacts: complete metal-silicate equilibration and core merging without any significant equilibration. They claimed that the observed isotopic data require re-equilibration of impacting bodies with the target mantle and ruled out direct core merging even for the largest impacting bodies. Although the direct core merging is one type of imperfect equilibration, it is not the only possible way of imperfectness. The Rayleigh-Taylor instability discussed in Section 2 is another type of imperfect equilibration, which leaves some fraction of silicate without equilibration with iron, while direct core merging leaves some fraction of iron without equilibration with silicate. Here we showed that the observational data does not rule out incomplete equilibration due to the Rayleigh-Taylor instability. Our results indicate that the collision conditions and the number of giant impacts are essential parameters to estimate the age of the core formation event.

\section{Discussion}

We have shown that complete metal-silicate equilibration by a giant impact cannot be expected even if the impact 
causes the whole mantle to become fully molten. We also showed that the age estimate varies dramatically, depending on the resetting ratio and the number of giant impacts, and that the resetting ratio of each giant impact is required to be greater than 0.2 on average. In the fully molten mantle situation, the equilibrated region (silicate-metal mixture layer) and non-equilibrated region (metal-free silicate melt layer) could overturn in a very short time, as shown in Fig. 3. If metal in the mixture layers (Fig. 3) becomes finely dispersed as small droplets, as described by Rubie et al. (2003), then only in these layers can the equilibration between metal and silicate be complete. An initially metalfree mantle, however, could not be equilibrated, because the overturn between the mixture layer and metal-free layer must occur before the metal droplets are able to sink significantly through the metal-free layer. This indicates that the volume of the initially well mixed metal-silicate layer resulting from each giant impact should be more than twotenths of the volume of the protoearth's mantle.

Such a mixture layer may correspond to the impactor's core, mantle, and the target's ejecta layer produced by a giant impact. Therefore, whether or not the mixture layer is well mixed and perfectly equilibrated between metal and silicate depends on how the target and impactor break, melt, vaporize, and accrete after a giant impact. In addition, SPH simulations of giant impacts suggest that the collision conditions of giant impacts greatly affect how the bodies break up. We therefore consider that the Hf-W chronometer also reflects collision conditions, such as the initial inward velocity and incident angle of each impactor.

Giant impacts would also have some important consequences for metal-silicate equilibration, including the partial vaporization of the target's mantle and possible breaking not only of the impactor's core but also of the target's core. At the very high temperatures just after a giant impact, some part of the target's upper layer of mantle could vaporize and mix effectively with the impactor's metal. Such vaporizations would enhance metal-silicate equilibration in the upper layer; however, the vaporization of the deep mantle is unlikely because of its very high pressure. A giant impact could break the target's core and distribute iron droplets in the mantle. These droplets could then equilibrate some part of the mantle; however, unless the iron droplets are evenly distributed throughout the whole mantle, the Rayleigh-Taylor instability will again prevent the equilibration of the whole mantle. Therefore, even in this case, perfect equilibration would only occur under very limited conditions, such as the complete vaporization of the target's mantle by a giant impact or the distribution of material from a broken target core throughout the whole metal-free layer of the mantle.

It is well known that the observed mantle abundance of moderately siderophile elements such as $\mathrm{Ni}$ and $\mathrm{Co}$ is larger than that predicted from low-pressure partitioning experiments (Ringwood, 1966). On the other hand, high-pressure experimental studies have shown that the high pressure and temperature partitioning can resolve this problem (e.g. Li and Agee, 2001). As shown below, our description of quick overturn of the mixture layer shown in Fig. 3 is consistent with a high pressure and temperature equilibrium between

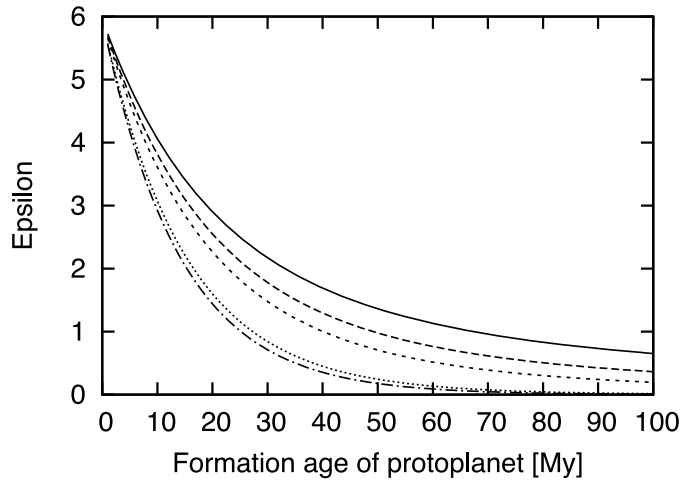

Fig. 9. Isotopic evolution of $\epsilon$ as a function of protoplanet formation age in the Mars region. Parameter $k$ [Eq. (6)] is $0.1,0.5,1,5,10$ from top to bottom.

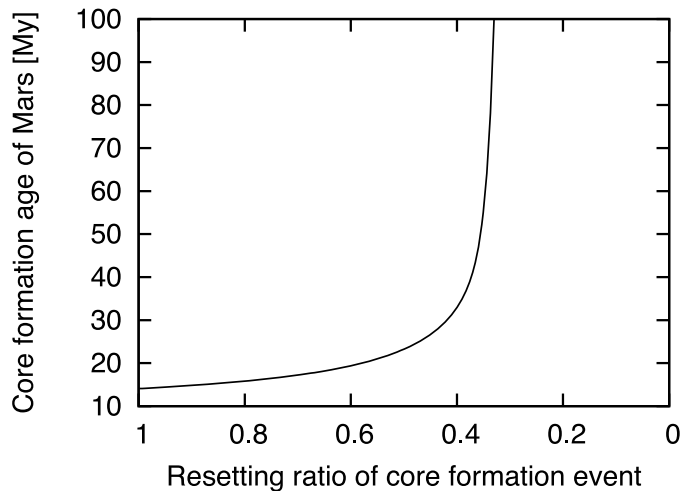

Fig. 10. The age of the core formation event of Mars as a function of the resetting ratio, fitting to the observational data $(\epsilon=2$, lowest value observed in SNC meteorites).

metal and silicate for moderately siderophile elements in a magma ocean scenario (e.g. Righter et al., 1997). Figure 3 shows that the segregated mixture layer including liquid metal droplets is deposited at the base of the molten mantle. Since the growth of the Rayleigh-Taylor instability of the mixture layer is very fast, equilibration between metal droplets and the silicate mantle occurs after the mixture layer has settled down, i.e., metal-silicate equilibration is expected to occur under high pressure at the base of the molten mantle. Each giant impact brings about the overturn of the molten mantle, and so multiple giant impacts could mix the molten mantle many times. Unlike tungsten, other siderophile elements are not produced by the decay of other elements. After multiple giant impacts, almost all siderophile elements except $\mathrm{W}$ in the mantle are equilibrated with metal under high pressure at the base of the molten mantle. Thus, the abundance of these elements is likely controlled by high-pressure equilibration.

We also considered the core formation event of Mars from the viewpoint of Hf-W chronometry. We calculated the isotopic evolution of $\epsilon$ in the Mars region using Eq. (6) with $f=2$ (less than the protoearth's value: $f=12$ ). This value was chosen because Mars is more oxidizing than the Earth. W does not efficiently partition into the core, and so the Hf/W ratio for the bulk silicate of Mars should be lower than that of the Earth (Kong et al., 1999; Kleine et 
al., 2002). The result is shown in Fig. 9. The result shows $\epsilon=3-4$ at $10 \mathrm{Myr}$, which is slightly larger than the $\epsilon$ value for Mars $[\epsilon=2$, the lowest value observed in SNC meteorites, (Lee and Halliday, 1997; Kleine et al., 2002, 2004a; Foley et al., 2005)], with a timescale of growth of protoplanets from planetesimals of $10^{5}-10^{6}$ years in the terrestrial planet region (Kokubo and Ida, 2000). This indicates that Mars could have experienced some sort of equilibrating event after the formation of the protoplanets and result is consistent with Nimmo and Agnor (2006), who implied that Mars may not simply be a stranded planetary embryo. We calculated the isotopic evolution for Mars in the same way as for the Earth. Figure 10 shows the age of core formation of Mars as a function of the resetting ratio of the core formation event, fitting the lowest $\epsilon$-value observed in Martian meteorites. This result indicates that the resetting ratio of the core formation event of Mars is required to be greater than 0.3. Therefore, Mars could have experienced an event that equilibrated more than three-tenths the volume of Mars' mantle, such as a single giant impact.

\section{Conclusions}

To achieve perfect equilibration, the iron droplets must be less than meter-scale, which makes the sedimentation velocities of the droplets low. The sedimentation velocity is therefore much slower than the growth of the RayleighTaylor instability between the upper, metal-containing silicate layer and the lower, metal-free silicate layer. In other words, if a metal-free layer exists in the first place, its equilibration is inhibited by the overturn of metal-containing and metal-free layers. When the equilibration is imperfect, the collision conditions and the number of giant impacts must be known in order to estimate the age of core formation of the Earth. Nevertheless, measurements of the tungsten isotope of terrestrial and SNC meteorites can provide constraints on giant impact events on Earth and Mars.

Acknowledgments. We thank Q.-Z. Yin for many helpful suggestions and comments, and M. Ozima and S. Sugita for valuable discussions. We also thank T. Kleine and another anonymous reviewer for their constructive comments, which led us to greatly improve this paper. This study was completed under the support of a JSPS Research Fellowship.

\section{Appendix A.}

First we evaluate the characteristic growth time of the Rayleigh-Taylor instability for the (1) thick mixture layer and (2) thin mixture layer.

(1) Thick mixture layer: Suppose a fluid layer with thickness $b$ and density $\rho_{1}$ (metal grains + silicate melt) lies on top of another fluid layer with the same thickness $b$ but with density $\rho_{2}$ (silicate melt), with $\rho_{1}>\rho_{2}$. Both fluid layers have the same viscosity $\eta$. The upper boundary of the top layer and the lower boundary of the bottom layer are assumed to be rigid surfaces. This assumption gives slower growth of the Rayleigh-Taylor instability than occurs in the free surface case. We can therefore estimate the upper limit (longer limit) of the growth time. The growth time of a disturbance $\tau_{r t 1}$ depends on the wavelength $\lambda$ of the interface displacement (Turcotte and Schubert, 2001)

$$
\begin{aligned}
& \tau_{r t 1}=\frac{4 \eta}{\left(\rho_{1}-\rho_{2}\right) g b} \cdot \frac{\theta^{2}+\theta \cosh \theta \sinh \theta}{\sinh ^{2} \theta-\theta^{2}}, \\
& \theta=\frac{2 \pi b}{\lambda}
\end{aligned}
$$

When the heavy fluid lies on top, the disturbance with the shortest time constant grows and dominates the instability. The wavelength that gives the smallest value for $\tau_{r t 1}$ is

$$
\lambda=2.568 b
$$

The growth time of the fastest growing disturbance is obtained by substituting Eq. (A.2) into Eq. (A.1) with the result

$$
\tau_{r t 1}=\frac{13.04 \eta}{\left(\rho_{1}-\rho_{2}\right) g b}
$$

(2) Thin mixture layer: Suppose a fluid layer with thickness $b$ and density $\rho_{1}$ lies on top of the semi-infinite fluid layer with density $\rho_{2}\left(\rho_{1}>\rho_{2}\right)$. Both fluid layers have the same viscosity $\eta$ and both upper and lower boundaries are free because the upper layer is assumed to be a thin layer. We can derive the relationship between the growth time of a disturbance $\tau_{r t 2}$ and the wavelength $\lambda$ of the interface distortion with the same procedures as for situation (1).

The relationship between physical values at the surface of the upper layer $(z=H$; subscript number 1$)$ and the boundary between two layers ( $z=0$; subscript number 2 ) is described below:

$$
\begin{aligned}
& {\left[\begin{array}{l}
W_{2} \\
U_{2} \\
S_{2} \\
F_{2}
\end{array}\right]=} \\
& {\left[\begin{array}{cccc}
-\theta \sinh \theta+\cosh \theta & \theta \cosh \theta & \theta \sinh \theta & -\theta \cosh \theta+\sinh \theta \\
-\theta \cosh \theta & \theta \sinh \theta+\cosh \theta & \theta \cosh \theta+\sinh \theta & -\theta \sinh \theta \\
-\theta \sinh \theta & \theta \cosh \theta+\sinh \theta & \theta \sinh \theta+\cosh \theta & -\theta \cosh \theta \\
-\theta \cosh \theta+\sinh \theta & \theta \sinh \theta & \theta \cosh \theta & -\theta \sinh \theta+\cosh \theta
\end{array}\right]} \\
& {\left[\begin{array}{c}
W_{1} \\
U_{1} \\
S_{1} \\
F_{1}
\end{array}\right]}
\end{aligned}
$$

where $W, U$ are velocities, $S$ is shear stress, and $F$ is surface traction. $\theta$ is the dimensionless layer thickness. We applied the following boundary conditions: (1) shear stress is zero and surface fraction corresponds to the surface displacement at $z=H$; (2) the velocities and shear stress are continuous and the surface fraction corresponds to the surface displacement at $z=0$. Therefore,

$$
\begin{aligned}
& S_{1}=U_{1} \\
& S_{2}=0 \\
& F_{2}=-\tau W_{2} \\
& F_{1}=(1+\delta \tau) W_{1}
\end{aligned}
$$


It is then possible to derive the following equation

$$
\begin{aligned}
A \delta \tau^{2}+ & \left(B_{1} \delta+B_{2}\right) \tau+C=0 \\
A & =\cosh \theta \sinh \theta-\theta+\sinh ^{2} \theta \\
B_{1} & =\cosh ^{2} \theta-\theta^{2}+\theta+\cosh \theta \sinh \theta \\
B_{2} & =\cosh ^{2} \theta+\sinh ^{2} \theta+2 \cosh \theta \sinh \theta \\
C & =(\cosh \theta+\sinh \theta)^{2} \\
\delta & =\frac{\rho_{2}-\rho_{1}}{\rho_{1}}
\end{aligned}
$$

which can be solved to derive

$$
\begin{aligned}
\tau_{r t 2}= & \frac{\eta \theta}{\rho_{1} g b} \\
& \cdot \frac{B_{1} \delta+B_{2}}{A \delta}\left[\sqrt{1-\frac{4 A B_{2} \delta}{\left(B_{1} \delta+B_{2}\right)^{2}}}-1\right]
\end{aligned}
$$

On the assumption that the lower layer is semi-infinite, higher order terms of $\theta$ can be assumed to be negligible and Eq. (A.15) is expanded:

$$
\tau_{r t 2}=\frac{\left(\sqrt{2} \rho_{1}^{2}-\sqrt{2} \rho_{1} \rho_{2}+4(\sqrt{2}+1) \rho_{2}^{2}\right) \eta}{\rho_{1} \rho_{2}\left(\rho_{1}-\rho_{2}\right) g b}
$$

The density of the upper layer, $\rho_{1}$ is obtained by

$$
\rho_{1}=\frac{1}{V_{\mathrm{si}}+V_{\text {metal }}} \cdot\left\{\rho_{\mathrm{si}} \cdot V_{\mathrm{si}}+\rho_{\text {metal }} \cdot V_{\text {metal }}\right\}
$$

where $V_{\mathrm{si}}, V_{\text {metal }}$ and $\rho_{\mathrm{si}}, \rho_{\text {metal }}$ represent volumes and densities of the silicate melt and impactor's core, respectively. Typical values are $\rho_{\mathrm{si}}\left(=\rho_{2}\right)=4 \times 10^{3} \mathrm{~kg} / \mathrm{m}^{3}$, and $\rho_{\text {metal }}=$ $12 \times 10^{3} \mathrm{~kg} / \mathrm{m}^{3}$.

Next we evaluated the characteristic sedimentation time of metal grains by Stokes sedimentation. According to Eq. (1), the timescale $\tau_{s}$ for an iron sphere to sink a distance of $l$ by Stokes sedimentation is given by

$$
\tau_{s}=\frac{l}{v_{s}}=\frac{9 \eta l}{2 \Delta \rho_{s} g r^{2}}
$$

Thus, we can compare the timescales of Stokes sedimentation and the growth of the Rayleigh-Taylor instability using Eq. (A.3), Eq. (A.16), and Eq. (A.18).

$$
\begin{aligned}
\frac{\tau_{r t 1}}{\tau_{s}} & =\frac{26.08 \cdot \Delta \rho_{s} \cdot r^{2}}{9 \cdot\left(\rho_{1}-\rho_{2}\right) \cdot b l} \\
\frac{\tau_{r t 2}}{\tau_{s}} & =\frac{2 \cdot\left(\sqrt{2} \rho_{1}^{2}-\sqrt{2} \rho_{1} \rho_{2}+4(\sqrt{2}+1) \rho_{2}^{2}\right) \Delta \rho_{s} \cdot r^{2}}{9 \cdot \rho_{1} \rho_{2}\left(\rho_{1}-\rho_{2}\right) \cdot b l}
\end{aligned}
$$

Assuming that $b$ in Eq. (A.19) is $1.45 \times 10^{6} \mathrm{~m}$, the half depth of the present Earth's mantle, and $b$ in Eq. (A.20) is very small, e.g. $1 \times 10^{4} \mathrm{~m}$, we can estimate the ratio $\tau_{r t 1} / \tau_{s}$ and $\tau_{r t 2} / \tau_{s}$.

\section{References}

Cameron, A. G. W., The Origin of the Moon and the Single Impact Hypothesis V, Icarus, 126, 126-137, 1997.
Cameron, A. G. W., Higher-Resolution Simulations of the Giant Impact, in Origin of the Earth and Moon, edited by R. M. Canup and K. Righter, 133-144, Arizona Press, 2000.

Canup, R. M., Simulations of a Late Lunar-Forming Impact, Icarus, 168, 433-456, 2004

Canup, R. M. and E. Asphaug, Origin of the Moon in a Giant Impact Near the End of the Earth's Formation, Nature, 412, 708-712, 2001.

Elsasser, W. M., Early History of the Earth, in Earth Science and Meteorites, edited by J. Geiss and E. Goldberg, 1-30, North-Holland Pub. Co., 1963.

Foley, C. N., M. Wadhwa, L. E. Borg, P. E. Janney, R. Hines, and T. L. Grove, The early differentiation history of Mars from ${ }^{182} \mathrm{~W}-{ }^{142} \mathrm{Nd}$ isotope systematics in the SNC meteorites, Geochim. Cosmochim. Acta, 69, 4557-4571, 2005.

Goodman, J., Convective Instability of Hollow Sedov-Taylor Blast Waves, ApJ, 358, 214-228, 1990.

Halliday, A. N., Mixing, Volatile Loss and Compositional Change During Impact-Driven Accretion of the Earth, Nature, 427, 505-509, 2004.

Halliday, A., M. Rehkämper, D.-C. Lee, and W. Yi, Early Evolution of the Earth and Moon: New Constraints from Hf-W Isotope Geochemistry, EPSL, 142, 75-89, 1996.

Harper, C. L., Jr. and S. B. Jacobsen, Evidence for ${ }^{182} \mathrm{Hf}$ in the Early Solar System and Constraints on the Timescale of Terrestrial Accretion and Core Formation, Geochim. Cosmochim. Acta, 60, 1131-1153, 1996.

Harper, C. L., Jr., J. Völkening, K. G. Heumann, C.-Y. Shih, and H. Wiesmann, ${ }^{182} \mathrm{Hf}^{-}{ }^{1} 82 \mathrm{~W}$ : New Cosmochronometric Constraints on Terrestrial Accretion, Core Formation, the Astrophysical Site of the r-Process, and the Origin of the Solar System, Lunar Planet. Sci. 22. Abstract, 515, 1991.

Iga, K. and R. Kimura, Bubble Convection, Central Core of the Earth, 3, 275-298, 1993.

Jacobsen, S. B., Isotopic and Chemical Constraints on Mantle-Crust Evolution, Geochim. Cosmochim. Acta, 52, 1341-1350, 1988.

Jacobsen, S. B., The Hf-W Isotopic System and the Origin of the Earth and Moon, Annu. Rev. Earth Planet. Sci., 33, 531-570, 2005.

Jacobsen, S. B. and C. L. Harper, Jr., Accretion and Early Differentiation History of the Earth Based on Extinct Radionuclides, in Earth Processes: Reading the Isotopic Code, edited by A. Basu and S. Hart, $47-$ 74, AGU, 1996.

Kleine, T., C. Münker, K. Mezger, and H. Palme, Rapid Accretion and Early Core Formation on Asteroids and the Terrestrial Planets from HfW Chronometry, Nature, 418, 952-955, 2002.

Kleine, T., K. Mezger, H. Palme, and C. Münker, The W Isotope Evolution of the Bulk Silicate Earth: Constraints on the Timing and Mechanisms of Core Formation and Accretion, EPSL, 228, 109-123, 2004a.

Kleine, T., K. Mezger, C. Münker, H. Palme, and A. Bischoff, ${ }^{182} \mathrm{Hf}-$ ${ }^{182} \mathrm{~W}$ isotope systematics of chondrites, eucrites, and martian meteorites: Chronology of core formation and early mantle differentiation in Vesta and Mars, Geochim. Cosmochim. Acta, 68, 2935-2946, 2004b.

Kleine, T., K. Mezger, H. Palme, E. Scherer, and C. Münker, Early core formation in asteroids and late accretion of chondrite parent bodies: Evidence from $182 \mathrm{Hf}-182 \mathrm{~W}$ in CAIs, metal-rich chondrites, and iron meteorites, Geochim. Cosmochim. Acta, 69, 5805-5818, 2005.

Kobayashi, S., Y. Abe, and Y. Fukao, Fluid Motion Induced by Gravitational Differentiation of Immiscible Two Phases: Basic Equations and Linear Analyses, J. Geomag. Geoelectr., 45, 1467-1480, 1993.

Kokubo, E. and S. Ida, Oligarchic Growth of Protoplanets, Icarus, 131 171-178, 1998.

Kokubo, E. and S. Ida, Formation of Protoplanets from Planetesimals in the Solar Nebula, Icarus, 143, 15-27, 2000.

Kong, P., M. Ebihara, and H. Palme, Siderophile Elements in Martian Meteorites and Implications for Core Formation in Mars, Geochim. Cosmochim. Acta, 63, 1865-1875, 1999.

Lee, D.-C. and A. N. Halliday, Hafnium-Tungsten Chronometry and the Timing of Terrestrial Core Formation, Nature, 378, 771-774, 1995.

Lee, D.-C. and A. N. Halliday, Hf-W Isotopic Evidence for Rapid Accretion and Differentiation in the Early Solar System, Science, 274, 18761879,1996

Lee, D.-C. and A. N. Halliday, Core Formation on Mars and Differentiated Asteroids, Nature, 388, 854-857, 1997.

Li, J. and C. B. Agee, The Effect of Pressure, Temperature, Oxygen Fugacity and Composition on Partitioning of Nickel and Cobalt between Liquid Fe-Ni-S Alloy and Liquid Silicate: Implications for the Earth's Core Formation, Geochim. Cosmochim. Acta, 65, 1821-1832, 2001.

Müller, E., B. Fryxell, and W. D. Arnett, High Resolution Numerical Simulations of Instabilities, Mixing, and Clumping in Supernova 1987A, 
in Proc. of ESO/EIPC Workshop on SN1987A and Other Supernovae, edited by I. J. Danziger and K. Kjär, 99, 1991.

Newsom, H. E., K. W. W. Sims, P. D. Noll, Jr., W. L. Jaeger, S. A. Maehr, and T. B. Beserra, The depletion of tungsten in the bulk silicate earth: Constraints on core formation, Geochim. Cosmochim. Acta, 60, 11551169, 1996.

Nimmo, F. and C. B. Agnor, Isotopic outcomes of N-body accretion simulations: Constraints on equilibration processes during large impacts from Hf/W observations, EPSL, 243, 26-43, 2006.

Oddershede, L., P. Dimon, and J. Bohr, Self-Organized Criticality in Fragmenting, Phys. Rev. Lett., 71, 3107-3110, 1993.

Righter, K., M. J. Drake, and G. Yaxley, Prediction of Siderophile Element Metal-Silicate Partition Coefficients to $20 \mathrm{GPa}$ and $2800^{\circ} \mathrm{C}$ : the Effects of Pressure, Temperature, Oxygen Fugacity, and Silicate and Metallic Melt Compositions, Phys. Earth Planet. Int., 100, 115-134, 1997.

Ringwood, A. E., Chemical Evolution of the Terrestrial Planets, Geochim. Cosmochim. Acta, 30, 41-104, 1966.

Rubie, D. C., H. J. Melosh, J. E. Reid, C. Liebske, and K. Righter, Mechanisms of Metal-silicate Equilibration in the Terrestrial Magma Ocean, EPSL, 205, 239-255, 2003.

Sasaki, S. and K. Nakazawa, Metal-Silicate Fractionation in the Growing
Earth: Energy Source for the Terrestrial Magma Ocean, J. Geophys. Res., 91, 9231-9238, 1986.

Sasaki, T. and Y. Abe, The effect of partial resetting on Hf-W system by giant impacts, in 36th ISAS Lunar and Planet. Symp. Proc., 21-24, 2003.

Schönberg, R., B. S. Kamber, K. D. Collerson, and O. Eugster, New Wisotope evidence for rapid terrestrial accretion and very early core formation, Geochim. Cosmochim. Acta, 17, 3151-3160, 2002.

Stevenson, D. J., Models of the Earth's Core, Science, 214, 611-619, 1981.

Turcotte, D. L. and G. Schubert, Geodynamics, Cambridge, 2001.

Vockenhuber, C. et al., New Half-Life Measurement of ${ }^{182} \mathrm{Hf}$ : Improved Chronometer for the Early Solar System, Phys. Rev. Lett., 93, 1725011-172501-4, 2004.

Wehterill, G. W. and G. R. Stewart, Accumulation of a Swarm of Small Planetesimals, Icarus, 77, 330-357, 1989.

Yin, Q.-Z., S. B. Jacobsen, K. Yamashita, J. Blichert-Toft, P. Télouk, and F. Albarède, A Short Timescale for Terrestrial Planet Formation from Hf-W Chronometry of Meteorites, Nature, 418, 949-952, 2002.

T. Sasaki (e-mail: takanori@eps.s.u-tokyo.ac.jp) and Y. Abe 